\title{
Development of a Thermographic Imaging Technique for Simultaneous Interfacial Temperature and Velocity Measurements
}

\author{
Alexandros Charogiannis, Ivan Zadrazil, Christos N. Markides \\ Clean Energy Processes (CEP) Laboratory, Department of Chemical Engineering, Imperial College London \\ London SW7 2AZ, United Kingdom \\ ac1005@ic.ac.uk; i.zadrazil06@imperial.ac.uk; c.markides@imperial.ac.uk
}

\begin{abstract}
An experimental technique, hereby referred to as 'thermographic particle velocimetry' (TPV) and capable of recovering twodimensional (2-D) surface temperature and velocity measurements at the interface of multiphase flows is presented. The proposed technique employs a single infrared (IR) imager and highly reflective, silver-coated particles, which when suspended near or at the interface, can be distinguished from the surrounding fluid due to their different emissivity. The development of TPV builds upon our previous IR imaging studies of heated liquid-film flows; yet, the same measurement principle can be applied for the recovery of 2-D temperature- and velocity-field information at the interface of any flow with a significant density gradient between two fluid phases. The image processing steps used to recover the temperature and velocity distributions from raw IR frames are demonstrated by application of TPV in a heated and stirred flow in an open container, and include the decomposition of each raw frame into separate thermal and particle frames, the application of perspective distortion corrections and spatial calibration, and the implementation of standard particle image velocimetry algorithms. Validation experiments dedicated to the measurement of interfacial temperature and velocity were also conducted, with deviations between the results generated from TPV and those from accompanying conventional techniques not exceeding the errors associated with the latter. Finally, the capabilities of the proposed technique are demonstrated by conducting temperature and velocity measurements at the gas-liquid interface of a wavy film flow downstream of a localised heater.
\end{abstract}

Keywords: Thermographic particle velocimetry (TPV), Infrared (IR) thermography, particle image velocimetry (PIV), particle tracking velocimetry (PTV), Liquid-film flows

\section{Introduction}

Liquid-film flows are convectively unstable open-flow systems endowed with a rich variety of wave regimes and hydrodynamic characteristics [1]. Ever since the Kapitza brothers [2] conducted the first pioneering experiments on the dynamics of gravity-driven falling films, hundreds of theoretical [3], numerical [4] and experimental [5] efforts have been devoted to the understanding of the physical processes behind these flows. Such efforts are still ongoing, fuelled by a strong desire to harness the heat and mass transfer capabilities of thin-film flows in a wide range of engineering and industrial applications. Examples of such applications include cooling schemes used in electronic and mechanical systems, heat exchangers, film condensers, evaporators and reactors, to name but a few.

In the case of locally or uniformly heated falling films (i.e. under non-isothermal conditions), the interface topology is strongly influenced by Marangoni stresses induced by surface tension gradients. The latter arise from temperature nonuniformities over the gas-liquid interface and are responsible for destabilizing the flow and triggering several well-studied phenomena such as film contraction, rivulet formation and film rupture. In search for increasing insight into these phenomena and aimed at the provision of highly detailed space- and time-resolved information on the interfacial characteristics of falling films, contemporary experimental investigations have largely relied on the application of optical diagnostic techniques. For example, planar laser-induced fluorescence (PLIF), which involves illumination and imaging of a planar section through the flow of interest, allows for spatiotemporally resolved film-height measurements [6-8], while particle image velocimetry (PIV) and particle tracking velocimetry (PTV) can be used for 2-D or 3-D flow-field characterization [9, 10]. In the latter case, sequential illumination and imaging of the scattered or fluorescence signal from particles seeded in the flow allows for tracking of the motion of particle groups (PIV) or individual particles (PTV), typically using a multi-pass cross-correlation approach during image post-processing. Infrared (IR) thermography has also been employed [7, 8], as it allows for temperature measurements to be performed over an extended, 2-D spatial domain with very high sensitivity (typically around $20 \mathrm{mK}$ ) and accuracy (often better than $0.5^{\circ} \mathrm{C}$ ), at relatively high repetition-rates (of the order of $100 \mathrm{~Hz}$ ). It should finally 
be noted that the increased popularity of IR imaging in the field non-isothermal film flow investigations can also be attributed to the fact that liquids such as water and glycerol, which are commonly employed in such studies, are opaque over broad IR spectral bands, allowing for temperature measurements that are intergraded over extremely shallow optical depths (of the order of a few 10 s of $\mu \mathrm{m})$.

The effects of Marangoni-induced stressed on wave topology in response to uniform [11], as well as localized heating [12], have been investigated by numerous researchers at the Institute of Thermophysics of the Siberian Branch of the Russian Academy of Sciences. By employing IR thermography, the particular group has contributed several noteworthy advances in our understanding of the link between the applied heat flux and the formation and spatiotemporal evolution of film regions dominated by thermal rivulets. Z.-B. Zhang and his co-workers $[13,14]$ have also conducted several thermal imaging studies on heated falling films, mainly focusing on film contraction/expansion phenomena, as a function of both flow parameters and heat input to the flow. Finally, the interaction between thermocapillary forces and the hydrodynamics of regular (solitary) wave patterns have been investigated by researchers at the Institute of Heat and Mass Transfer of Aachen University [15, 16]. In that case, IR thermography was employed alongside a film-height measurement technique in order to elucidate the role of the Marangoni effect on film dynamics. Before closing this brief introduction, it should be noted that the techniques and contributions cited here are only indicative of the considerable number of intriguing studies carried out by employment of optical diagnostics in falling-film flows.

In this contribution we propose an alternative approach to the use of basic IR imaging for the study of heated fallingfilm flows. Building upon our recent work with unsteady and conjugate heat transfer in thin liquid-film flows [7, 8], and the experimental investigation of the hydrodynamic characteristics of isothermal films by simultaneous application of PLIF and PIV/PTV [10], we seek to extend our understanding of the phenomenology of the formation and evolution of thermal features on the film free-surface and to investigate the coupling between these features and the hydrodynamic characteristics of heated falling films, by simultaneously conducting thermography and velocimetry measurements on the gas-liquid interface [17]. The proposed experimental technique aims to achieve this by employing highly reflective, silver-coated particles, which when suspended near or at the gas-liquid interface, are imaged as highly localized thermal radiation peaks (Fig. 1 (a)) or troughs (Fig. 1 (b)) due to their differing emissivity characteristics compared to the surrounding fluid.

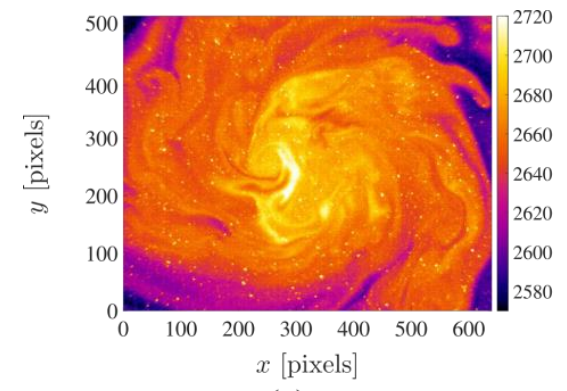

(a)

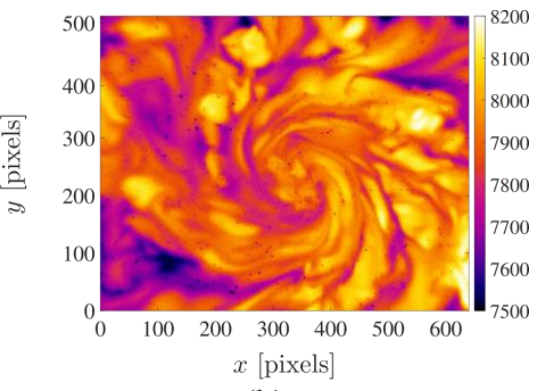

(b)

Fig. 1: (a) Raw IR images of heated, particle-laden flows at the gas-liquid interface of a heated and stirred flow of water inside an open cylindrical container. (a) Images taken with the water temperature being lower than the background temperature and the reflective particles appearing as localized glare spots. (b) Images taken with the water temperature being higher than the background temperature and the particles appearing as localized dark spots.

\section{Experimental Methodology}

The thermal radiation collected by the IR camera, $I_{\text {meas, }}$ when the latter is positioned over the gas-liquid interface follows the relation given in Eq. (1):

$$
I_{\text {meas }}=\epsilon I_{\mathrm{li}}-(1-\epsilon) I_{\mathrm{bg}}
$$

Where $\epsilon$ stands for the emissivity of the liquid, $I_{\mathrm{li}}$ for the thermal radiation emitted by the liquid, and $I_{\mathrm{bg}}$ for the background radiation reflected by the liquid as a result of its reflectivity $\rho=1-\epsilon$. The emissivity of water, the liquid employed in our experiments, amounts to 0.96 [18]; in contrast, the emissivity of silver, the material used to coat the hollow glass 
particles is below 0.1 even when oxidized. Thus, the apparent temperature of the liquid is indicative of its real temperature, while that of the particles is largely dictated by the background radiation.

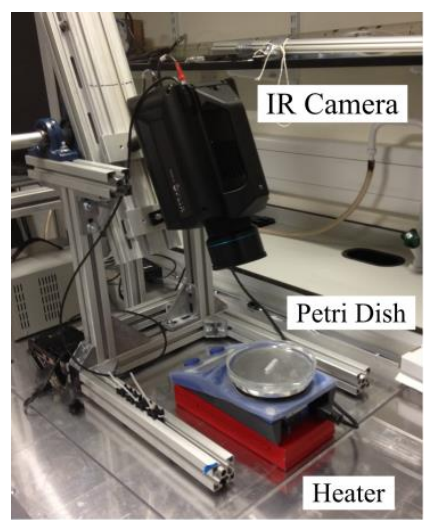

(a)

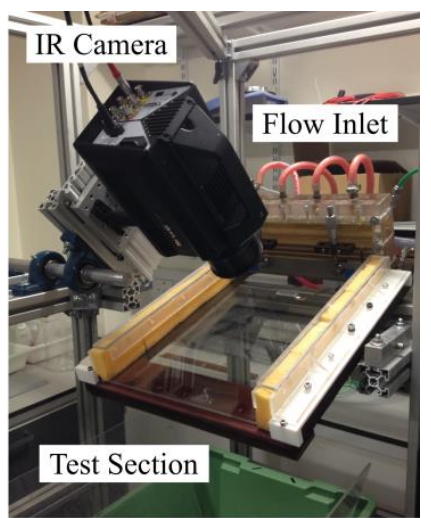

(b)

Fig. 2: Photographs of the experimental arrangements used (a) for developing the experimental methodology and processing routines, and validating the IR-based temperature measurement, and (b) in the velocity validation and localized heating experiments. The images presented in Fig. 1 were generated using the arrangement shown in Fig. 2(a).

In developing the experimental methodology and associated image processing steps, a simple experimental setup was employed (Fig. 2 (a)). A cooled, mid-wave FLIR X6540sc camera $(640 \times 520$ pixel detector, thermal sensitivity of approximately $18 \mathrm{mK}$ and maximum recording frequency of $126 \mathrm{~Hz}$ ) was positioned over a heated and stirred flow of water inside an open container seeded with silver-coated glass hollow spheres (100 $\mu \mathrm{m}$ mean diameter, $12 \%$ silver content by weight, $0.9 \mathrm{~g} / \mathrm{cc}$ density) purchased from Hart Materials Ltd. The particles were selected to be buoyant/neutral in order to allow for interfacial velocity measurements with only modest seeding concentrations. The camera was equipped with a 25 $\mathrm{mm}, \mathrm{F} / 2$ lens (transmissive over the range $2.5-5 \mu \mathrm{m}$ ), allowing for a maximum spatial resolution of up to around 100 $\mu \mathrm{m} / \mathrm{pixel}$, while the image integration time was set to $843 \mu \mathrm{s}$. The flow was generated using a $650 \mathrm{~W}$ IKA RCT magnetic stirrer with intergraded temperature control. A raw thermal image of the liquid surface with the particle-seeded water heated up to approximately $60^{\circ} \mathrm{C}$ and continuously stirred at $100 \mathrm{rpm}$ is shown in Fig. 3 (a).

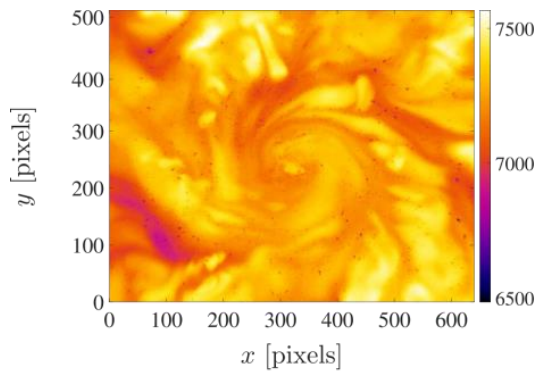

(a)

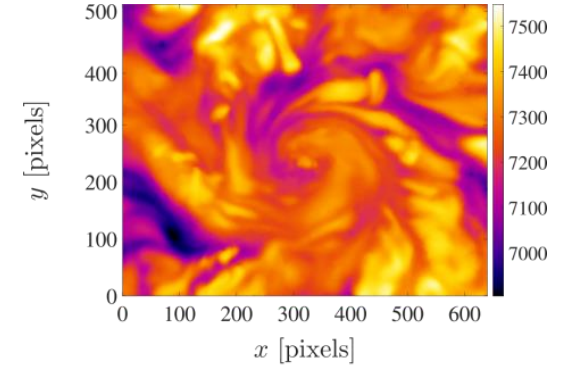

(b)

Fig. 3: Raw thermal images of the heated and stirred flow with (a) the particles present in the flow and (b) with the particles removed during postprocessing. The colour-scales next to the images indicate the intensity of the collected raw signal.

In this experiment the camera axis was set at an angle of approximately $20^{\circ}$ to the flow in order to prevent it from capturing an image of itself, and consequently, perspective distortion corrections were implemented later on during processing using a thermal target image of known spatial coordinates. The target itself comprised a black carton background and a perforated and polished steel sheet $(4.7 \times 4.7 \mathrm{~mm}$ rectangular holes, $8 \mathrm{~mm}$ pitch). Following the collection of thermal images, the latter were processed in Matlab using an in-house developed algorithm. Firstly, the particles were identified in the original frames by subtracting a $3 \times 3$-pixel moving average, and two separate frames were generated; one containing the particles and a second image of the flow with the particles removed. The former was also subjected to a threshold intensity level determined a priori by conducting experiments without particles in the flow, in order to remove any noise and minimize 
inaccuracies in the PIV calculation (Fig. 4 (b)). Once the particle-only frame was fully processed, it was used to mask out the pixels corresponding to particles in the thermal frame, and the latter was reconstructed by substituting those values with the average of their neighbours. Both thermal and particle frames were then imported into LaVision Davis 8.2 and corrected for perspective distortions using a pinhole model. The resulting apparent resolution corresponded to $110 \mu \mathrm{m} / \mathrm{pixel}$ in this particular experiment. The perspective-distortion corrected thermal frames were finally imported back into MATLAB and converted to temperature maps using the IR camera calibration (Fig. 4 (a)).

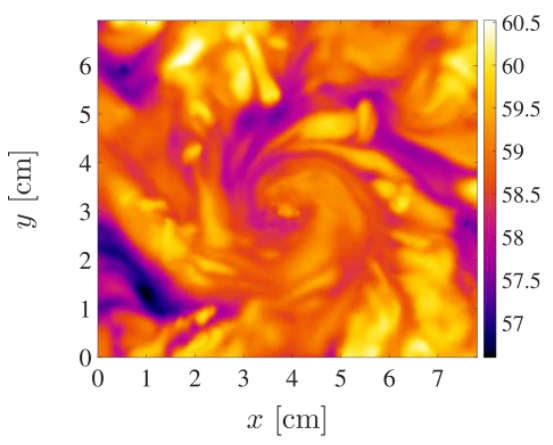

(a)

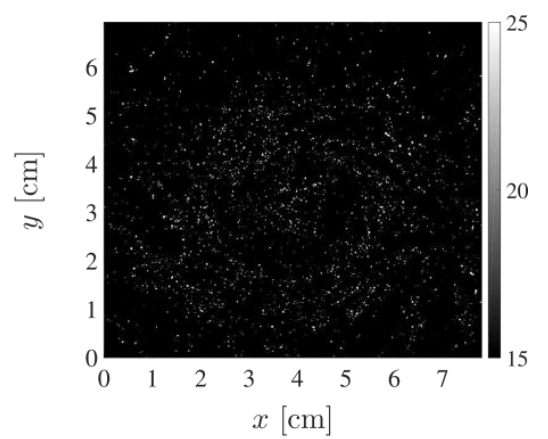

(b)

Fig. 4: (a) Perspective-distortion corrected temperature, and (b) particle frames generated during the intermediate processing steps of temperature and velocity field calculation. The colour-scale next to frame (a) indicates the liquid temperature and the one next to frame

(b) the intensity of the particle signal following the moving average subtraction.

The particle frames were processed using a four-pass cross-correlation with a $64 \times 64$ pixel interrogation window and $50 \%$ overlap for the first two passes, and a $32 \times 32$ pixel window in the final two passes, resulting in a $1.7 \mathrm{~mm}$ vector-tovector spatial resolution. Individual particles were also tracked (PTV calculation) using the PIV results as reference estimators of the velocity field. The PTV and PIV velocity-vector maps obtained from Fig. 4 (b) were overlaid with the temperature field (Fig. 4 (a)) and are presented in Fig. 5 (a) and (b), respectively.

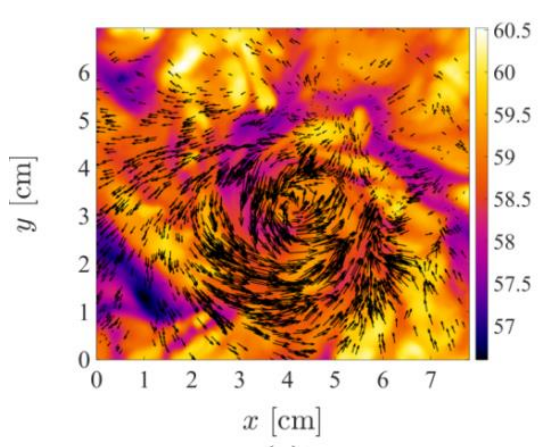

(a)

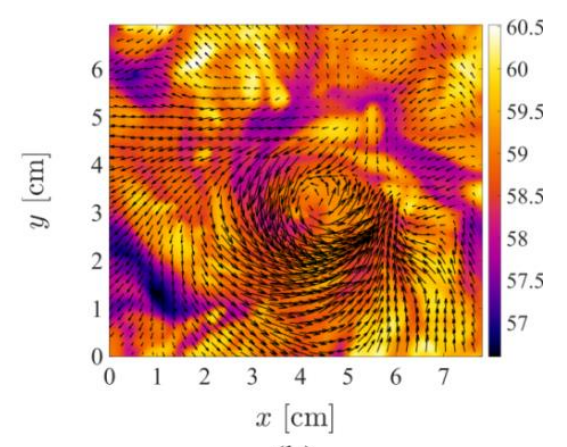

(b)

Fig. 5: (a) Combined temperature and PTV, and (b) temperature and PIV measurements, generated based on the particle image presented in Fig. 4(b) and the temperature field in Fig. 4(a). The colour-scales next to each frame indicate the liquid temperature.

\section{Validation Experiments}

\subsection{Temperature Measurement Validation}

Having developed and optimized the processing routine using the simple setup described earlier, temperature and velocity validation experiments were performed, the former using the same setup and the latter by employment of the fallingfilm apparatus described in Refs. [7, 8, 10] and shown in Fig. 2 (b). In the first case, temperature measurements were recorded with the IR camera positioned over the flow and with pre-calibrated K-type thermocouples arranged around the periphery of the Petri dish (Fig. 6 (a)). With the liquid pre-heated to $80^{\circ} \mathrm{C}$, the heater was turned off and the flow was continuously stirred while cooling down to room temperature $\left(25^{\circ} \mathrm{C}\right)$. Based on the IR camera recordings, the temperature standard deviation at the gas-liquid interface varied between $0.5{ }^{\circ} \mathrm{C}$ and $2.5{ }^{\circ} \mathrm{C}$ over the entire imaging region, depending on the flow mean 
temperature (higher at higher mean temperatures). Therefore, only a limited $(1.5 \mathrm{~cm} \times 1.5 \mathrm{~cm})$ region of interest (ROI) was considered for each thermocouple for the purposes of this test (the four regions corresponding to the four thermocouples are shown in Fig. 6 (a)). Finally, the IR camera temperature measurements $\left(T_{\mathrm{IR}}\right)$ were plotted against the thermocouple measurements $\left(T_{\mathrm{TC}}\right)$ in Fig. 6 (b). The error bars represent the maximum measurement error associated with the thermocouple measurements, which according to the manufacturer, amounts to $2.5{ }^{\circ} \mathrm{C}$. The deviation between the IR camera and thermocouple results was below $\pm 0.5^{\circ} \mathrm{C}$ at a $64 \%$ confidence level, and below $\pm 1{ }^{\circ} \mathrm{C}$ at an $80 \%$ confidence level over the examined temperature range. The latter is a representative value of the maximum error associated with the IR temperature measurement.

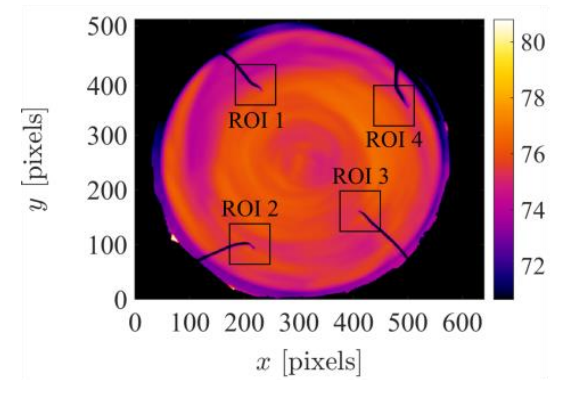

(a)

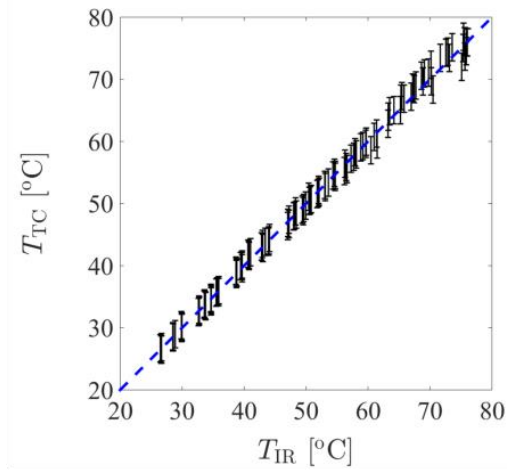

(b)

Fig. 6: (a) Temperature validation experiment conducted using four K-type thermocouples and an IR camera, the latter set up to image the gas-liquid interface of a continuously stirred, cooling water volume in an open container. (b) Temperature measurements, $T_{\mathrm{IR}}$, conducted using the IR camera, plotted against temperature measurements, $T_{\mathrm{TC}}$, recovered using thermocouples, over the range $25-75{ }^{\circ} \mathrm{C}$.

\subsection{Velocity Measurement Validation}

The validity of the proposed interfacial velocity measurement technique was assessed by conducting tests in the falling-film apparatus (Fig. 2 (b)), which comprises a planar test-section over which film flows develop, a settling chamber installed at the test section inlet, and a closed flow-loop that incorporates a pump and a heat exchanger. In the absence of interfacial waves, the velocity profile across the liquid film can be obtained analytically using the 1-D, steady, fully developed solution of the Navier-Stokes equation for a gravity-driven flow, also referred to as the Nusselt solution (Eq. 2). The velocity at the interface is then given by:

$$
U_{\mathrm{Nu}}=\frac{1}{2}\left(\frac{g \sin \beta}{v_{\mathrm{f}}}\right)^{1 / 3}\left(\frac{3 Q}{w}\right)^{2 / 3}
$$

Where $\beta$ is the inclination angle (here set to $30^{\circ}$ to the horizontal), $v_{\mathrm{f}}$ the fluid kinematic viscosity, $Q$ the flow rate and $w$ the film span $(290 \mathrm{~mm})$. In order to generate results close to this prediction using the film rig, the growth of interfacial instabilities was moderated by selecting a high glycerol content ( $81 \%$ glycerol, $19 \%$ water by weight), resulting in a highly viscous aqueous-glycerol solution. The flow Reynolds number $(R e)$ was varied in the range $R e=0.6-11.8$ by adjusting the liquid flow-rate in the range $Q=0.11 \times 10^{-4}-1.28 \times 10^{-4} \mathrm{~m}^{3} \mathrm{~s}^{-1}$, and the kinematic viscosity in the range $v_{\mathrm{f}}=3.75 \times 10^{-4}-$ $6.42 \times 10^{-4} \mathrm{~m}^{2} \mathrm{~s}^{-1}$, the latter by modulating the temperature of the employed aqueous-glycerol solution between $19.3{ }^{\circ} \mathrm{C}$ and $28.3^{\circ} \mathrm{C}$. The liquid temperature was measured inside the settling chamber using a K-type thermocouple; based on this measurement and the known water/glycerol content, the density and kinematic viscosity were calculated using the parametrization provided in [19].

As these measurements were performed under isothermal conditions, the particles were illuminated using a $150 \mathrm{~W}$ IR heater, thus increasing the contrast with which they appeared on the thermal images relative to the liquid. It should be noted that separate thermocouple measurements were carried out in order to assess the effect of radiative heating on the film, with the temperature difference between runs with the heater on and off being lower than $1{ }^{\circ} \mathrm{C}$ even for $\operatorname{Re}<0.8$. Thus, it can be ascertained that this practice did not affect the measurement. Finally, the transmittance of glycerol over the spectral band 2.5 
- $5 \mu \mathrm{m}$ was measured using an FT-IR spectrometer (Perkin-Elmer Spectrum 100) for path lengths ranging between 60 and $1040 \mu \mathrm{m}$. Our results indicate that the penetration depth corresponds to approximately $60 \mu \mathrm{m}$, and therefore, interfacial temperature measurements can be performed in flows containing high concentrations of glycerol, as the latter is effectively opaque.

The results of the velocity validation experiments are presented in Fig. 7(b), with a typical PIV map shown in Fig. 7 (a). For the purpose of this test, only a two-pass cross-correlation was employed, with the interrogation window size increased to $128 \times 128$ pixels and the overlap set to $50 \%$. These parameters were chosen on the basis that the measured flowfield only involves negligible spanwise velocity gradients, and thus, a reduced number of passes and a smaller window size would allow for faster processing with minimal inaccuracy. The dotted line in Fig. 7(b) corresponds to $U_{\exp }=U_{\mathrm{Nu}}$, the error bars indicate the range of $U_{\exp }$ values that lie within the experimental error associated with the calculation of $U_{\mathrm{Nu}}$, and the extent of the error bars corresponds to average errors that arise when using the analytical expression presented in Eq. (2) and used to generate estimates of $U_{\mathrm{Nu}}$. The mean deviation between the experimentally derived interfacial velocities and the analytically calculated ones based on the Nusselt solution was only around $2 \%$ over the range of velocities $0.05-0.3 \mathrm{~m} \mathrm{~s}^{-1}$. The latter is a representative value of the error associated with the PIV measurement. This experiment also demonstrates the prospective employment of the proposed technique in isothermal film flows, whereby interfacial velocities can be recovered over an extended spatial domain.

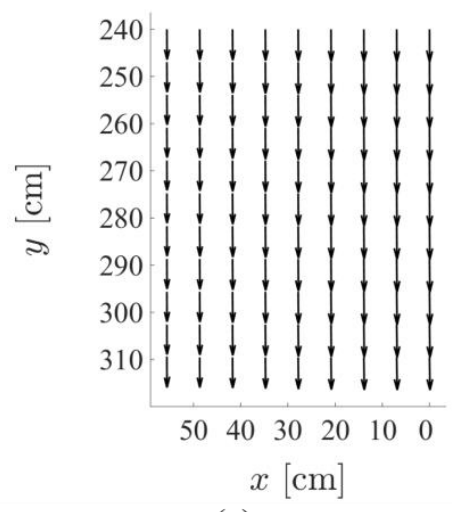

(a)

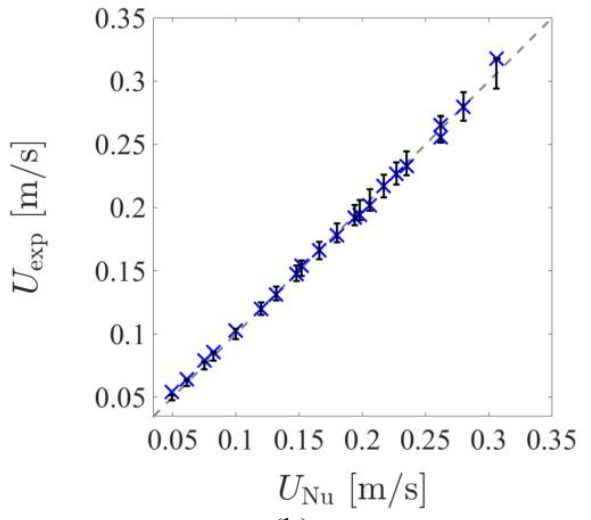

(b)

Fig. 7: (a) Interfacial PIV measurement carried out in a viscous film pertaining to $R e=11.8$. (b) Interfacial velocity measurements $U_{\text {exp }}$ extracted from interfacial PIV maps such as the one presented in (a), plotted against analytically calculated velocities values $U_{\mathrm{Nu}}$, for viscous films in the range $R e=0.6-11.8$.

\section{Film Flow Experiments}

The falling-film setup was modified in order to demonstrate the combined thermographic velocimetry technique in a non-isothermal film-flow experiment. In greater detail, the glass substrate was replaced by a perspex one with a $4 \times 1.3 \mathrm{~mm}$ groove cut across the direction of the flow at a distance of $7.5 \mathrm{~cm}$ from the flow inlet. The groove was centred relative to the film span. A rectangular metal sleeve housing a $200 \mathrm{~W}$ cartridge heater was installed in the groove and powered by a programmable $6 \mathrm{~kW}$ power supply (Magna-Power Electronics Ltd). The water-glycerol solution was replaced with water which was cooled down to $17.5^{\circ} \mathrm{C}$, the substrate inclination angle was reduced to $15^{\circ}$ (to the horizontal), and the power input to the cartridge heater was set to $44 \mathrm{~W}$. For the particular power input, the heat flux into the liquid corresponds to $9 \pm$ $0.3 \mathrm{~W} \mathrm{~cm}^{-2}$. Thermal images were recorded between approximately 5 and $9 \mathrm{~cm}$ downstream of the heater for water flows with $R e=80,100$ and 120, following the procedure outlined earlier, while particle images were processed using a four-pass cross-correlation, with the final window size set to $48 \times 48$ pixels and the overlap to $50 \%$. The corresponding vector-tovector resolution was $2.6 \mathrm{~mm}$.

Fully-processed sample images of the simultaneously recovered temperature and velocity fields are presented in Fig. 8 , revealing complex thermal features and highly inhomogeneous velocity distributions. In the case of the $R e=120$ film, three pronounced thermal 'rivulets' are observed along the direction of the flow, with shorter and thinner ones emerging in between the latter. The corresponding velocity vector map shows a fast-moving wave (Fig 8(e)) which coincides with the inception region of the dominant thermal structures. With reduced inertia $(\operatorname{Re}=100)$, the rivulets become more intertwined, 
most probably due to enhanced mixing. Reducing the $R e$ even further $(R e=80)$ brings about a considerable augmentation of the particular effect, with the rivulet formation zone moving further upstream (closer to the heater). The observed thermal field is now dominated by highly irregular thermal structures.

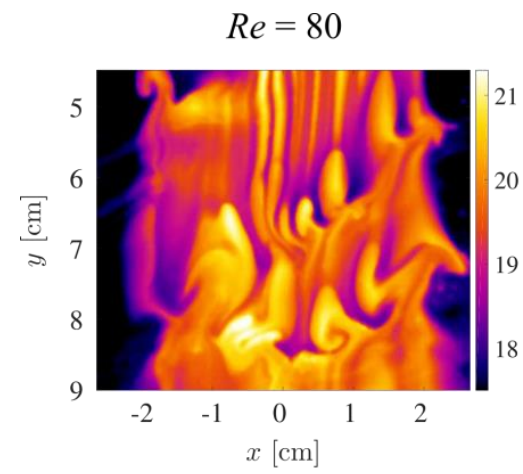

(a)

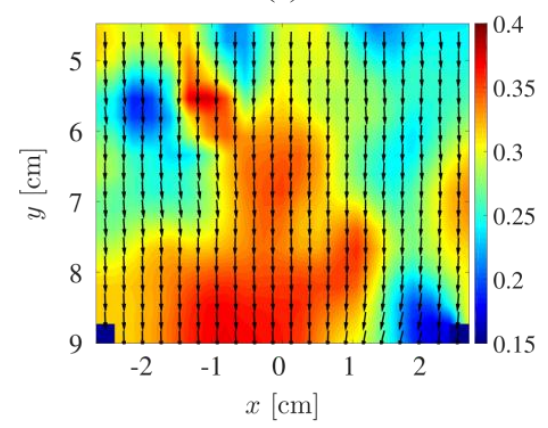

(d)

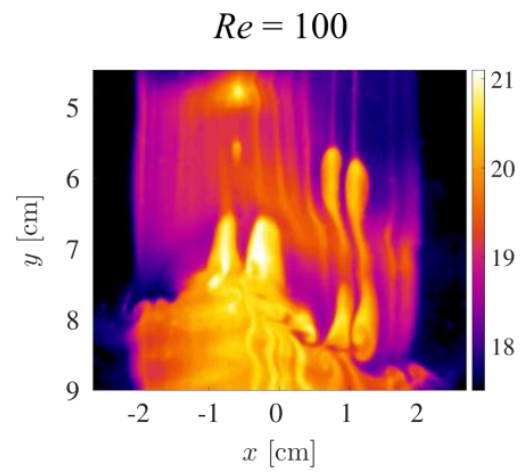

(c)

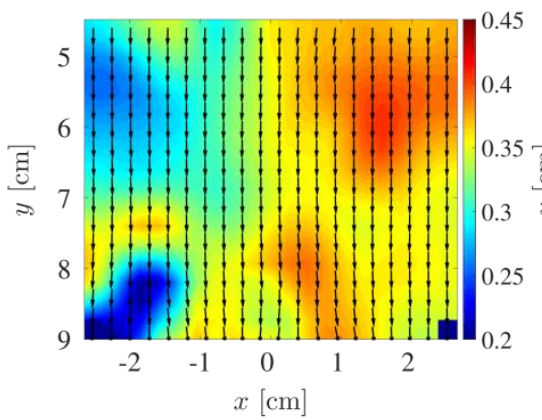

(f)

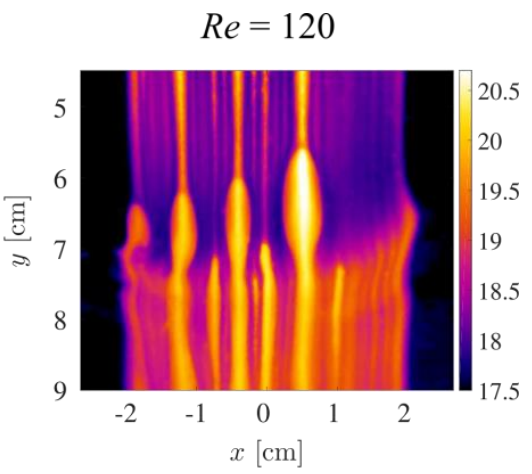

(b)

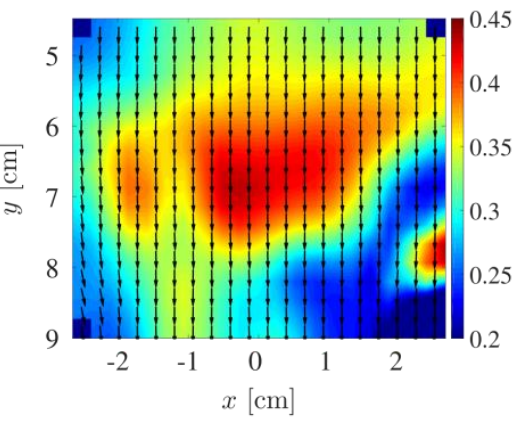

(e)

Fig. 8: Simultaneously measured interfacial temperature (top row) and velocity (bottom row) distributions downstream of localised heating element in falling water-films with $R e=80,100$ and 120. The y-axes indicate the distance of the imaging region from the heating element, while the colour-scales next to the PIV maps correspond to the magnitude of the local velocity.

\section{Conclusions}

The development, validation and application of a thermographic particle velocimetry (TPV) technique were presented in this contribution. The technique, which seeks to simultaneously recover two-dimensional (2-D) surface temperature and velocity measurements at the interface of multiphase flows, is based on the use of a single IR imager and highly reflective, silver-coated particles. The latter, when suspended near or at the interface, can be distinguished from the surrounding fluid due to their different emissivity. First, the image processing steps used to recover the temperature and velocity distributions from raw IR frames were demonstrated by application of TPV in a heated and stirred flow in an open container, and include the decomposition of raw thermal images into separate thermal and particle frames, the application of perspective distortion corrections and spatial calibration, and the implementation of standard particle image velocimetry algorithms. In addition to the experiments conducted for developing this methodology, validation experiments dedicated to the measurement of interfacial temperature and velocity were also presented, with deviations between the results generated from TPV and those from accompanying conventional techniques (thermocouple measurements and analytical predictions, respectively) not exceeding the errors associated with the latter. Thus, the errors associated with the TPV temperature- and velocitymeasurements do not exceed $3 \%$ and $2 \%$ respectively. Finally, temperature and velocity measurements were carried out at the gas-liquid interface of a wavy film flow downstream of a localised heater, in order to demonstrate the capabilities of the proposed technique in a typical film-flow investigation. These experiments reveal complex thermal features and highly inhomogeneous velocity distributions.

\section{Acknowledgements}

This work was supported by the UK Engineering and Physical Sciences Research Council (EPSRC) [grant number 
EP/K008595/1]. The authors would also like to acknowledge the valuable contribution of Manish Mehta, Dennis Choo and Khemmathin Lueangwattanapong.

\section{References}

[1] S. Kalliadasis, C. Ruyer-Quil, B. Scheid, and M. G. Velarde, Falling liquid films, Springer Series on Applied Mathematical Sciences, 2012.

[2] P. L. Kapitza and S. P. Kapitza, "Wave flow on thin layers of a viscous fluid," J. Exp. Theor. Phys., vol. 19, pp 105$120,1949$.

[3] S. Kalliadasis, E. A. Demekhin, C. Ruyer-Quil, and M. G. Velarde, "Thermocapillary instability and wave formation on a film falling down a uniformly heated plane," J. Fluid Mech., vol. 492, pp. 303-338, 2003.

[4] G. M. Skotheim, U. Thiele, and B. Scheid, "On the instability of a falling film due to localized heating," J. Fluid Mech., vol. 475, pp. 1-19, 2003.

[5] S. V. Alekseenko, V. Y. Nakoryakov, and B. G. Pokusaev, "Wave formation on a vertical falling liquid film," AIChE J., vol. 31, no. 9, pp. 1446-1460, 1985.

[6] I. Zadrazil, O. K. Matar, and C. N. Markides, "An experimental characterization of downwards gas-liquid annular flow by laser-induced fluorescence: Flow regimes and film statistics," Int. J. Multiph. Flow, vol 60, pp. 87-102, 2014.

[7] R. Mathie, H. Nakamura, and C. N. Markides, "Heat transfer augmentation in unsteady conjugate thermal systems Part II: Applications," Int. J. Heat Mass Transfer, vol. 56, pp. 819-833, 2012.

[8] C. N. Markides, R. Mathie, and A. Charogiannis, "An experimental study of spatiotemporally resolved heat transfer in thin liquid-film flows falling over an inclined heated foil," Int. J. Heat Mass Transfer, vol. 93, pp. 872-888, 2015.

[9] I. Zadrazil and C. N. Markides, "An experimental characterization of liquid films in downwards co-current gas-liquid annular flow by particle image and tracking velocimetry," Int. J. Multiph. Flow, vol. 68, pp. 1-12, 2014.

[10] A. Charogiannis, J. S. An, and C. N. Markides, "A simultaneous planar laser-induced fluorescence, particle image velocimetry and particle tracking velocimetry technique for the investigation of thin liquid-film flows," Exp. Therm. Fluid Sci., vol. 68, pp. 516-536, 2015.

[11] E. A. Chinnov, E. N. Shatskii, and O. A. Kabov, "Evolution of the temperature field at the three-dimensional wave front in a heated liquid film," High Temp., vol. 50, no. 1, pp. 98-105, 2012.

[12] E. A. Chinnov and S. S. Abdurakipov, "Thermal entry length in falling liquid films at high Reynolds numbers," Int. J. Heat Mass Transfer, vol. 56, pp. 775-786, 2013.

[13] F. Zhang, X. Zhao, J. Geng, Y.-T. Wu, and Z.-B. Zhang, "A new insight into Marangoni effect in non-v isothermal falling liquid films," Exp. Therm. Fluid Sci., vol. 31, no. 4, pp. 361-365, 2007.

[14] F. Zhang, J. Peng, J. Geng, Z.-X. Wang, and Z.-B. Zhang, "Thermal imaging study on the surface wave of heated falling liquid films," Exp. Therm. Fluid Sci., vol. 33, no. 3, pp. 424-430, 2009.

[15] V. V. Lel, A. Kellermann, G. Dietze, R. Kneer, and A. N. Pavlenko, "Investigations of the Marangoni effect on the regular structures in heated wavy liquid films," Exp. Fluids, vol. 44, no. 2, pp. 341-354, 2008.

[16] M. Rietz, W. Rohlfs, R. Kneer, and B. Scheid, "Investigations of the Marangoni effect on the regular structures in heated wavy liquid films,” Eur. Phys. J. Spec. Top., vo. 224, pp. 355-368, 2015.

[17] O. A. Kabov, B. Scheid, I. A. Sharina, and J.-C. Legros, "Heat transfer and rivulet structures formation in a falling thin liquid film locally heated," Int. J. Therm. Sci., vol. 41, no. 7, pp. 664-672, 2002.

[18] M. Q. Brewster, Thermal Radiative Transfer and Properties. Wiley-Interscience, 1992.

[19] N.-S. Cheng, "Formula for the viscosity of a glycerol water mixture," Ind. Eng. Chem. Res., vol. 47, no. 9, pp. 3285$3288,2015$. 